Revue de l'Institut des langues et cultures

d'Europe, Amérique, Afrique, Asie et Australie

$16 \mid 2012$

La culture progressiste à l'époque de la guerre froide

\title{
Les Pièces de guerre d'Edward Bond : une approche politique de la guerre nucléaire hors des canons militants
}

David Tuaillon

\section{OpenEdition}

Édition électronique

URL : http://journals.openedition.org/ilcea/1399

DOI : 10.4000/ilcea.1399

ISSN : 2101-0609

Éditeur

UGA Éditions/Université Grenoble Alpes

Édition imprimée

ISBN : 978-2-84310-232-5

ISSN : 1639-6073

Référence électronique

David Tuaillon, «Les Pièces de guerre d'Edward Bond : une approche politique de la guerre nucléaire hors des canons militants », ILCEA [En ligne], 16 | 2012, mis en ligne le 04 juillet 2012, consulté le 22 mars 2021. URL : http://journals.openedition.org/ilcea/1399; DOI : https://doi.org/10.4000/ilcea.1399

Ce document a été généré automatiquement le 22 mars 2021

(C) ILCEA 


\title{
Les Pièces de guerre d'Edward Bond: une approche politique de la guerre nucléaire hors des canons militants
}

\author{
David Tuaillon
}

1 La guerre nucléaire n'a pas eu lieu, mais elle n'en a pas moins constitué un des emblèmes culturels les plus marquants de la guerre froide. Menace constante dans le réel et omniprésente dans les imaginaires, elle s'est imposée autant comme un objet de cristallisation politique que comme une problématique esthétique en soi. Nous nous proposons ici d'évoquer les Pièces de guerre (The War Plays), un cycle de trois pièces élaborées par Edward Bond entre 1983 et 1985, dont elle est le sujet. Il s'agit d'une proposition artistique singulière qui constitue une réponse culturelle à un aspect donné de la guerre froide dans un moment précis de son développement, et à laquelle nous ne prétendons pas accorder une valeur générale. En revanche, cette œuvre nous semble exemplaire d'une contribution majeure à l'avancée d'une culture progressiste ${ }^{1}$ permise par le contexte de la guerre froide. Nous commencerons par décrire brièvement ce contexte avant d'observer dans quelles conditions Bond conçoit et élabore son projet, puis nous étudierons les particularités de chaque pièce, pour enfin nous interroger sur leur réception.

2 Si la véritable probabilité d'une guerre nucléaire durant la première moitié des années 1980 reste sujette à débats historiques², il ne fait aucun doute que ce risque (et celui de l'anéantissement de toute la civilisation humaine qu'il sous-entendait ${ }^{3}$ ) ait été une préoccupation majeure des opinions publiques du monde développé à cette époque ${ }^{4}$. Sans nous étendre sur le faisceau de causes culturelles, politiques, et historiques qui soutient cette inquiétude, rappelons tout de même que ces dernières années de la guerre froide en sont les plus tendues et les plus dangereuses. Elles sont dominées par la crispation de chaque camp dans une position d'affrontement ouvert, qui trouve à se manifester très concrètement dans une accumulation de crises internationales, systématiquement gérées dans une logique de confrontation. 
3 La plus importante et la plus longue d'entre elles est la crise des Euromissiles, long bras de fer entre les deux blocs au sujet du renouvellement du parc de missiles nucléaires de portée intermédiaire en Europe, crise rendue encore plus aiguë par ses retombées dans la politique intérieure des États-membres de l'OTAN. Les opinions publiques se sont en effet emparées de ce débat, provoquant la résurgence d'un mouvement pacifiste de grande ampleur, appuyé par une authentique mobilisation citoyenne internationale de plusieurs années qui rassemble, à l'automne 1981, jusqu'à 3 millions de manifestants dans 90 villes européennes ${ }^{5}$. La Grande-Bretagne est l'une des nations les plus mobilisées, rassemblant des cortèges dont l'ampleur ne sera égalée que par ceux des opposants à la guerre en Irak de 2003. Ce mouvement est non seulement massif, mais très inventif dans ses formes, en partie parce que les acteurs culturels s'y impliquent en nombre, notamment les auteurs de théâtre. Tous les dramaturges actifs à l'époque écrivent en effet des pièces consacrées au risque de guerre nucléaire, qu'ils revendiquent toujours ouvertement comme un acte militant. Pour la plupart, ces œuvres se limitent cependant à relayer un seul discours de dénonciation, soit du cynisme des dirigeants, soit de la douleur des victimes de la guerre à venir. Jouant du pathétique ou du sarcasme, et produites pour manifester du consensus sur des convictions déjà partagées par le public, elles n'offrent guère d'approche critique ou d'analyses structurelles ${ }^{6}$. C'est dans ce contexte, aussi vif et virulent politiquement que pauvre et conventionnel esthétiquement, que peuvent s'évaluer les véritables innovations, en tant que théâtre politique, des Pièces de guerre.

4 En ce début d'années 1980, Edward Bond a déjà une œuvre théâtrale conséquente derrière lui et vient de connaître une décennie de grande reconnaissance publique et critique. Il n'est pas engagé dans les mouvements pacifistes mais il cherche à écrire sur la guerre nucléaire, sans trouver un juste point de vue pour le faire ${ }^{7}$. Depuis quelques années, à côté de son activité d'auteur et de metteur en scène, Bond dirige régulièrement des ateliers de théâtre avec des étudiants ou des élèves acteurs dans une perspective clairement politique. À partir de sujets d'actualité ou des discussions entre étudiants, il élabore des situations dramatiques et des thèmes d'improvisation, mettant en jeu leurs positions et leurs oppositions. Il s'agit pour lui simplement d'utiliser les moyens du théâtre pour rendre actifs les convictions et les arguments de tous, afin de les y impliquer personnellement et directement. C'est fut lors d'un de ces ateliers, qu'il dirige à l'université de Palerme à l'automne 1983, qu'il trouve la clef pour écrire sur la guerre nucléaire.

5 Le principal débat politique entre les étudiants palermitains à l'époque est justement l'installation en cours de 112 missiles Pershing dans la base de Comiso toute proche et l'opportunité de s'en servir, même préventivement, pour dissuader le Pacte de Varsovie d'utiliser les siens. Bond ramène les termes de ces débats à une question morale élémentaire (qui rejoint celle de la dissuasion) : Faut-il se préparer à tuer son ennemi avant qu'il ne nous tue ? ou plus précisément : Doit-on, pour protéger nos enfants, tuer les enfants des autres? Avec ces données, il compose une situation dramatique qu'il soumet à ses étudiants, en supprimant toutes les médiations pour mettre au jour les contradictions de leurs positions : au lieu de rester des citoyens qui votent pour des dirigeants qui ordonneront à des militaires d'appuyer sur un bouton pour projeter des missiles à des milliers de kilomètres qui tueront des enfants qu'ils n'auront jamais vus, Bond les met en situation d'être eux-mêmes un soldat recevant directement l'ordre de tuer l'enfant de leur voisin. Le prétexte de ce massacre des innocents contemporain est 
une situation de famine insurmontable où les enfants en bas âge, de toutes façons condamnés à court terme, ne seraient plus que des bouches inutiles. Bond propose plusieurs variantes à ce schéma pour limiter les échappatoires et les justifications externes ou ouvrir les possibilités d'approche, mais une constante demeure : le soldat doit agir dans sa rue où ne se trouve que deux bébés, celui de la voisine et celui de sa mère.

6 Tel que Bond le relate ${ }^{8}$, les étudiants lors du déroulement des improvisations renoncent tous à tuer l'enfant de la voisine comme on l'attend d'eux, puis retournent chez leur mère pour tuer, à sa place, leur petite sœur. La clef de cet étrange "paradoxe de Palerme », comme le baptise Bond, semble tenir au parallélisme exact entre les deux bébés, tous deux égaux et également innocents, qui dénonce, au-delà de l'horreur du meurtre, la logique inhumaine qui les désigne l'un victime et l'autre mobile d'un crime. Bond, avec cet exercice, avait pensé révéler aux participants leur implication personnelle et profonde dans une violence d'État dont ils réprouvent pourtant a priori la forme (à la manière de l'expérience de Milgram), mais il découvre au contraire une instance de résistance au fond de l'être humain. Il en tire le fil d'une réflexion très personnelle sur l'anthropologie théâtrale qu'il déroule encore aujourd'hui -sur laquelle nous ne nous étendrons pas ici. Cet événement lui fournit aussi la matrice des Pièces de guerre, non seulement parce que l'épisode improvisé par les étudiants lui donne sa trame, mais surtout parce que cette forme de résistance constitue la dynamique du propos des pièces, et leur permet de dépasser le discours d'indignation ambiant et sa logique victimaire compassionnelle.

7 À la suite de cet atelier, tout au long de l'année 1984, Bond écrit trois pièces, dans des circonstances autonomes, mais qu'il rassemble ensuite en un cycle homogène pour former les Pièces de guerre.

8 Rouge noir et ignorant (Red Black and Ignorant) la première, adopte la forme du théâtre d'agit-prop, c'est-à-dire du théâtre d'intervention militante tel qu'il se pratiquait alors le plus couramment ${ }^{9}$ - et dont relevaient de nombreuses pièces que nous évoquions plus haut ${ }^{10}$. Cependant, si la pièce en affiche tous les codes (visée démonstrative portée par une adresse directe, légèreté du dispositif impliquant un symbolisme élémentaire, théâtralité immédiate du jeu et commentaires autonomes), c'est en réalité pour les détourner, principalement grâce au personnage central que Bond appelle «le Monstre ». Il s'agit d'un enfant tué dans le ventre de sa mère par les bombardements nucléaires de la guerre qui arrivera très prochainement dans la vie réelle des spectateurs. Une indication liminaire le décrit ainsi: «sa peau, ses cheveux et ses vêtements sont carbonisés, entièrement noirs, comme taillés dans un morceau de charbon. Ses cheveux hérissés, des pointes raides, se dressent comme des clous». Une telle figure peut passer pour une allégorie comme on en trouve fréquemment dans l'agit-prop: outrancière et macabre, manifestée par des signes élémentaires mais frappants (masques, postiches, effets criards). Il s'agit en réalité d'une représentation naturaliste d'un corps calciné tel qu'on en voit sur les photographies des bombardements nucléaires de Hiroshima et Nagasaki ${ }^{11}$. Ce fait est d'autant plus frappant que l'aspect du Monstre est le seul élément désigné avec précision parmi un dispositif spartiate et allusive, limité à un banc à tout faire et un pain.

9 Le Monstre mène, littéralement, la pièce, s'adressant très directement aux spectateurs pour annoncer les scènes avant de les jouer puis de les commenter, rôle choral tout à fait classique dans l'esthétique de l'agit-prop, à ceci près qu'il passe en principe par la 
distanciation. Il revient d'habitude à un acteur qui ne se dissimule pas derrière le masque d'un personnage, ou se donne les moyens explicites de s'en détacher, et qui, en avant-garde éclairée, idéalement sorti des rangs du public, assume un commentaire sur une action dont il démontre la signification aux spectateurs. Ici, c'est une figure de fiction pleinement manifestée comme telle, un témoin légitime qui prend la parole ès qualités, pour montrer, sans s'extraire de sa fiction « des scènes de la vie qu'il n'a pas vécue ». Ainsi la fonction critique, classiquement conditionnée par le recours à la distanciation, sensée garantir l'objectivité de la perception dans l'esthétique brechtienne, est renversée pour être produite depuis l'intérieur de la fiction.

Cet enfant mort-né, arrivé du futur proche des spectateurs dont il est la victime promise, image du « citoyen inconnu » (pour reprendre le titre original de la pièce : The Unknown Citizen) de la guerre à venir, est aussi un équivalent pour ce prochain crime contre l'humanité de la figure du «témoin intégral» décrite par Primo Levi pour Auschwitz, ces Muselmänner qui «ne sont pas revenus pour raconter » mais qui auraient pu être les vrais témoins d'Auschwitz parce qu'ils ont éprouvé « la destruction menée à son terme, l'œuvre accomplie » des camps ${ }^{12}$. L'existence éphémère du Monstre, hors de toute expérience antérieure (puisqu'il n'est jamais né), témoigne toute entière de ce qu'est la condition humaine dans le moment de la destruction du monde par l'homme et le rend apte, lui et lui seul, à nous en dire la vérité. Et seule la fiction du théâtre, avec les torsions paradoxales qu'elle rend possibles, autorise ce spectre d'un homme ni encore né, ni déjà mort, à revenir pour nous parler, de plein droit avec ses moyens et ses prérogatives et faire valoir son expérience sur nous.

11 La position tenue par la pièce grâce à ce personnage arrache le spectateur au protocole compassionnel dans lequel la place habituellement le discours victimaire pour le placer devant ses responsabilités, accompagné par un témoin légitime qui le lui signifie sans ambages, notamment dans cet envoi qui clôt la pièce :

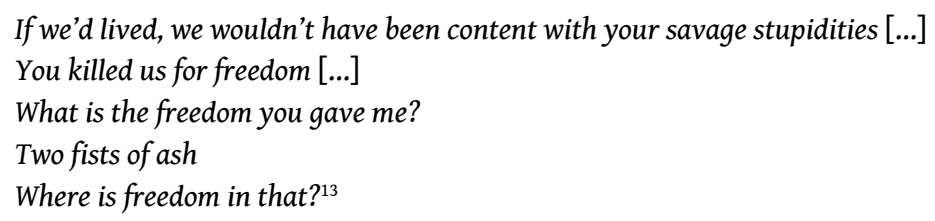

Bond écrit la deuxième Pièce de guerre, La Furie des nantis (The Tin Can People), pour une compagnie de théâtre militant, mais préfére à la forme attendue de l'agit-prop, la grande forme de la tragédie grecque, courant à la catastrophe dans une alternance d'épisodes et de chœurs et avec une certaine solennité poétique et rhétorique. La pièce porte encore plus loin le renversement du discours victimaire pour exposer un autre type d'analyse.

13 Nous sommes vingt ans après une guerre nucléaire globale et définitive qui a transformé le monde en un vaste désert stérile, mais dans ce lieu commun de la science-fiction, Bond développe une hypothèse moins attendue pour construire une métaphore politique. Il imagine un groupe d'enfants ayant réussi à survivre aux bombardements et qui se seraient rassemblés autour d'un gigantesque stock de nourritures de l'armée épargné par les bombes, sur lequel ils pourraient se nourrir éternellement. Bond invente là une société d'abondance post-nucléaire, qu'il qualifie de "société capitaliste la plus avancée " c'est-à-dire un état d'opulence illimitée et sans travail ${ }^{14}$. Cette communauté prospère et paisible n'est cependant pas une communauté heureuse. Ses membres doivent tout aux morts parmi lesquels ils ne constituent qu'une anomalie dérisoire, ils restent obsédés par les horreurs de la guerre, ne savent rien 
faire et ne font rien et sont très dépressifs. Par ailleurs, ils sont (significativement) stériles et donc condamnés à la disparition sans avoir rien produit.

La pièce met en scène la crise qui les saisit le jour où apparaît pour la première fois depuis dix-huit ans un étranger. Il est l'exact contraire de ces fainéants dans leur vallée stérile : il a survécu seul dans le désert, sans jamais cesser de marcher et de parler dans l'espoir de rencontrer un autre, travaillant ainsi toujours du corps et de l'imagination pour maintenir son humanité. Ils espèrent d'abord qu'il pourrait être leur premier père, avant de découvrir qu'il porte une maladie qui les tue un à un. Du moment où ils décident de se débarrasser de lui, nous assisterons au retour, étape par étape, de la violence à l'intérieur de cette société, dans les pensées, les discours et les actes : repli sanitaire, puis sécuritaire, justification légale du meurtre, organisation étatique, militaire, et finalement religieuse, jusqu'à ce que, rien n'étant parvenu à enrayer leur panique, ils basculent dans l'irrationnel achevé et détruisent toutes leurs réserves, déclenchant une guerre nucléaire à leur échelle. Cette grande émeute finale, une orgie de destruction intégrale, apparaît ainsi comme une pure dépense, dans le sens de Georges Bataille d'une "hécatombe des biens»: "forme improductive» de la consommation, qui manifeste et obéit à " un besoin de perte démesurée qui existe à l'état endémique dans un groupe social ${ }^{15} »$.

Les survivants de la pièce n'ont donc rien des misérables attendus qui envieraient les morts, mais sont au contraire une upper class, une haute bourgeoisie jouissant de privilèges exclusifs mais hantés par leur destruction. La pièce montre ainsi que les racines de la guerre nucléaire, son ethos, sont à chercher dans les fonctionnements même de la société capitaliste de consommation et non dans ses insuffisances marginales. La pièce s'achève sur une conclusion très classiquement marxiste, qui voit les survivants débarrassés de leur manne héritée des morts, prêts à se réaliser euxmêmes dans leur travail et par la propriété collective de leurs propres moyens de production et ainsi s'appartenir à eux-mêmes pour trouver par eux-mêmes le sens de leur existence en tant qu'individu et que communauté. Cette pièce aussi, donc, s'emploie davantage à une analyse structurelle des comportements sociaux qu'à une dénonciation idéologique et trouve la voie d'une position politique directe, même si ce n'était pas celle qu'on attendait.

La troisième pièce, Grande paix (Great Peace) ne relève plus du tout de l'économie de l'urgence des précédentes. C'est une pièce de grande ampleur nécessitant quatre heures de représentation, avec de nombreux personnages et un souffle épique mais relevant d'un mode de narration tout à fait classique. Bond y reprend l'improvisation de Palerme littéralement pour en observer les processus et les prolongements. Il situe l'action dans une ville soumise à la loi martiale après un bombardement nucléaire et l'ordre scélérat est autant une mesure sanitaire qu'autoritaire pour restaurer l'ordre, devenant ainsi un acte extrême de pure domination biopolitique.

Nous suivons le parcours du soldat, qui agit exactement comme à Palerme : il reçoit l'ordre, rentre chez lui, tente de tuer le bébé de la voisine mais y renonce, puis il retourne chez sa mère et finalement décide de tuer sa petite sœur. La pièce nous le montre aussi après l'infanticide : de retour dans son unité, il reste mutique et refuse obstinément d'exécuter un ordre sanitaire trivial (ramasser le paquet de cigarettes jeté par son sous-officier) jusqu'à être exécuté pour insubordination. Bond permet donc à ce personnage de prolonger son expérience au-delà du paradoxe pour insister sur l'aspect 
libératoire du paradoxe en montrant la possibilité d'une résistance intérieure à l'ordre - même si celle-ci reste symbolique.

La pièce s'intéresse cependant surtout au parcours de la mère du soldat qui en est en réalité le personnage principal. La Femme (telle que la désigne Bond) appartient au prolétariat conservateur (celui qui peu avant a fait élire Margaret Thatcher). C'est une brave dame et une bonne citoyenne, certaine de son droit et indignée par les désordres, qui bénéficie de la protection de l'armée où est engagé son fils - mais aussi de la nourriture qu'il détourne lors de perquisitions. Elle est par ailleurs baby-sitter et garde justement chez elle l'enfant de la voisine lorsque son fils vient la trouver et lui révèle la nature de ses ordres. Elle est d'abord choquée à l'extrême et refuse en bloc de coopérer, mais on la voit céder progressivement à l'urgence de la situation et sous la pression de son fils. Une fois qu'elle a admis que l'armée peut assassiner les enfants, elle accepte graduellement que son fils tue un enfant, mais à condition qu'il soit mourant, ou malade, ou seulement abandonné, puis finalement lui livre celui dont elle s'occupe tous les jours - à la seule condition qu'il ne le tue pas chez elle. Cette évolution ne s'arrête pas là puisque, lorsque le fils faillit à son discutable devoir, nous verrons la Femme tenter elle-même, pour sauver son enfant, d'assassiner de ses mains celui de la voisine. Ce crime (évité de justesse par un deus ex machina), puis le meurtre de son enfant qu'elle vit comme un châtiment surnaturel, lui font perdre la raison. À travers le comportement individuel de la Femme, Bond a ainsi retracé la position qu'il avait voulu critiquer dans l'improvisation de Palerme, celle des humanistes sincères, opposés à la violence mais qui l'acceptent malgré tout à la première inquiétude, si elle frappe un autre. Il force ici son personnage à en assumer personnellement la responsabilité.

Il n'est donc plus question dans cette pièce d'un discours de déploration. Bond n'en appelle ni à la compassion pour les victimes impuissantes, ni à la stigmatisation des coupables cyniques, mais énonce, dans la construction et l'analyse d'une situation, une critique en termes politiques de la participation des citoyens à des actes criminels de l'État alors qu'ils les réprouvent moralement. Position paradoxale et pourtant commune résumée par la Femme au moment où elle va se résoudre à devenir une criminelle, dans des termes aussi élémentaires que directs :

I know its wrong $t^{\prime}$ say these things but what else can I do? ${ }^{16}$

La pièce propose aussi la possibilité d'une reconstruction humaine après la catastrophe, qui passe par le sens de la communauté, énoncé en des termes remarquablement peu idéologiques. Sa seconde partie nous montre la Femme, vingt ans après la même fin du monde que dans La Furie des nantis, errant dans le désert et portant avec elle un baluchon qu'elle traite comme son enfant mort. Par une série de rencontres avec d'autres survivants, elle pourra, dans un processus symboliquement théâtral, reconstituer son histoire en rejouant les événements et les confrontations qui l'ont amenée à commettre l'intolérable pour comprendre son crime et celui de sa société. Elle achève la pièce par un geste paradoxal (encore un) mais qui se veut acte de fondation humaine pour une nouvelle colonie de survivants.

21 Conçues en réponse à une dynamique militante, dans un contexte historique et politique très déterminé, ces pièces sont donc parvenues à dépasser tant dans leur esthétique que dans leur propos, les carcans inhérents à ce champ de production qui entravaient le développement d'une analyse critique fondamentale nécessaire à l'établissement d'un véritable discours critique et politique sur la guerre nucléaire. 

elle aussi singulière et inattendue. Créées dans des conditions très médiocres à l'été 1985 par la Royal Shakespeare Company, celles-ci passent à peu près inaperçues en Grande-Bretagne et ne participèrent donc en rien aux débats qui leur ont donné naissance. Les Euromissiles étaient d'ailleurs désormais installés (les luttes pacifistes ne l'avaient pas empêché) et l'apaisement apporté par Mikhaïl Gorbatchev, qui venait d'arriver au pouvoir en Union soviétique, allait bientôt éloigner le spectre de la guerre nucléaire. Ces pièces ne trouveraient véritablement une audience que dans une tout autre situation : en France, en 1994, lors de leur création par Alain Françon au Festival d'Avignon. Les Pièces de guerre connaissent alors un grand succès et ont un fort impact sur les spectateurs, non seulement durant le Festival, toujours propice aux réceptions passionnées, mais aussi, fait plus rare, lors de sa reprise à l'odéon-Théâtre de l'Europe en janvier suivant. Cet événement réinstalle la figure de Bond dans le paysage théâtral français à la place de premier plan qu'il occupe toujours aujourd'hui.

Cette réception décalée dans l'espace et dans le temps est d'autant plus surprenante que la menace de la guerre nucléaire, désormais éloignée, est alors remplacée par une nouvelle actualité de la guerre, et avec elle un nouvel imaginaire, qui ne lui ressemble en rien : les guerres génocidaires au Rwanda et en ex-Yougoslavie. La première révèle justement son horreur en cet été 1994 et la seconde est une grande cause portée par les intellectuels et les artistes, notamment à Avignon ${ }^{17}$. Ainsi en ce début d'années 1990, de nombreux metteurs en scène affirment leur volonté (accompagnée sans doute d'une réelle demande) d'un discours contemporain sur la guerre, et cherchent pour le tenir des détours, en particulier par la caution, à la fois esthétique, politique et littéraire, de la tragédie athénienne $\mathrm{e}^{18}$. Ces intentions restent le plus souvent vaines : à la fois trop général et trop restreint par le phénomène immédiat, et peut-être aussi trop déclaré, ce sujet se dissout sans doute en cherchant à se cristalliser dans des figures qui lui sont éloignées.

Ce discours est justement pris en charge par les représentations des Pièces de guerre alors même que l'équipe de création ne le proclame pas du tout. L'image de la guerre qu'elles portent, déchargée de son actualité sans être pour autant effacée des mémoires, a désormais une portée métaphorique suffisamment large pour assumer un absolu, l'idée même de guerre, et n'est pas encore assez éloignée pour être devenue abstraite. La guerre nucléaire offre une allégorie matérielle, lisible comme image autant que comme expérience du monde, qui permet de traiter le sujet dans sa matière propre et non dans sa manifestation conjoncturelle. Entre l'anecdotique du présent et la généralité du mythe, le spectacle offre la possibilité moins d'une bonne distance, que d'une juste proximité de regard sur ce que le public de 1994 cherche à percevoir. Le spectacle se trouvait ainsi assumer une véritable position, dans le sens récemment défini par Georges Didi-Hubermann ${ }^{19}$, bien plus fondamentale qu'une colère du présent. Enfin, par l'ambition de son propos, la suggestion de sa fiction et la force de son verbe (assumées par la rigueur de ses interprètes et par sa durée: plus de huit heures, toute une nuit à Avignon), la représentation de Pièces de guerre se présente ouvertement comme une grande forme, mais actuelle, articulant de vastes questions dans les termes de notre temps et travaillant directement son sujet. Parce qu'elle est frontale, cette grande forme avec sa grande métaphore accomplit ce que d'autres avaient attendu d'un plus commode détour par la tragédie antique.

ILCEA, 16 | 2012 
ectacle reproduit ainsi, dans un contexte militant réactivé mais déplacé, et lui aussi impuissant à affronter son objet, le même effort qui avait été celui de Bond dix ans plus tôt, de chercher la position et le discours réellement critiques et politiques à tenir, ancré, mais dépassant le terrain qui lui donnait naissance.

\section{BIBLIOGRAPHIE}

BATAILLE Georges, « La Notion de dépense » [1933], dans La Part maudite, Paris, Éditions de Minuit, coll. « Critique », 1967.

Bond Edward, The War Plays, and Commentary on The War Plays, dans Plays, vol. 6, Londres, Methuen, 1998 (édition française : Pièces de guerre, trad. M. Vittoz, 2 vol., Paris, L'Arche, 1994 et Commentaire sur les Pièces de guerre et le paradoxe de la Paix, trad. G. Bas, Paris, L’Arche, 1994).

-, Selections from Edward Bond's Notebooks, éd. Ian Stuart, vol. 1 : 1959-1980, Londres, Methuen, 2000.

-, Poems (1978-1985), Londres, Methuen, 1987.

-, Letters, choisies et présentées par Ian Stuart, vol. 2, Luxembourg, Harwood Academic Publishers, 1995.

Didi-Hubermann Georges, Quand les images prennent position, L'œil de l'Histoire I, Paris, Éditions de Minuit, Paradoxe, 2008.

FRIEDMAN Norman, The Fifty-year War, Conflict and Strategy in the Cold War, Washington, US Naval Institute Press, 1999.

Katz Arthur M., Life After Nuclear War, The Social Impacts of Nuclear Attacks on the United States, Cambridge (MA), Ballinger Publishing, 1982.

LAUNAY Bertrand de, Le Poker nucléaire, comme brebis à l'abattoir, Paris, Syros, 1983.

LE GuELTE Georges, Les Armes nucléaires, mythes et réalités, Arles, Actes Sud, 2009.

LEVI Primo, I Sommersi E I Salvati, Milan, Enaudi, 1986 (Les Naufragés et les rescapés, Paris, Gallimard, 1989).

LowE Stephen, Peace Plays, 2 vol., Londres, Methuen, Theatrefile, 1985 \& 1990.

LOYER Emmanuelle et DE BAECQUE Antoine, Histoire du Festival d'Avignon, Paris, Gallimard, 2007.

LUECKEN Michael, 1945 - Hiroshima les images sources, Paris, Hermann Éditeur, 2008.

MiLgram Stanley, Obedience to Authority, An Experimental View, New York, Harpercollins, 1974

(Soumission à l'autorité, Paris, Calmann Lévy, 1994).

Neveux Olivier, Le Théâtre militant en France des années 1960 à nos jours, Paris, La Découverte, 2007.

SAGAN Carl, The Dark and the Cold, The World After Nuclear War, W. W. Norton \& Company, 1984 (Le Froid et les ténèbres, Paris, Belfond, 1985). 
ScHELl Jonathan, The Fate of the Earth, New York, Alfred A. Knopf, 1982 (Le Destin de la Terre, Paris, Albin Michel, 1982).

STUART Ian, Politics in Performance, the Production Work of Edward Bond, 1978-1990, New-York, Peter Lang Publishing, 1996.

Le Théâtre d'agit-prop de 1917 à 1932 (4 vol.) et Le Théâtre d'intervention depuis 1968, (2 vol.), Lausanne, L’Âge d'homme, coll. « Théâtre années 20 », 1977-1978 et 1983.

THOMPson James, Psychological Aspects of Nuclear War, Leicester, British Psychological Society, John Whiley \& Son, 1985.

\section{NOTES}

1. Nous adoptons ce terme d'autant plus aisément qu'il permet d'éviter les difficultés et les ambiguïtés qu'il y aurait à définir une culture « de gauche » en Grande-Bretagne.

2. Voir, par exemple, la récente synthèse de G. Le Guelte ou celle de N. Friedmann.

3. Les effets d'une guerre thermonucléaire globale ont été étudiés et décrits entre autres par J. Schell, A. M. Katz ou encore C. Sagan; ces textes étaient largement connus et relayés dans la presse.

4. À titre d'exemple, un sondage réalisé en Grande-Bretagne en 1982 indiquait que $72 \%$ des personnes interrogées se déclaraient «inquiètes" à l'idée d'une guerre nucléaire et $38 \%$ pensaient qu'elle aurait lieu de leur vivant (voir J. Thompson).

5. Pour un bilan chiffré et détaillé de ces mouvements à la fin de la vague, voir B. de Launay.

6. En témoigne, en particulier le recueil en deux volumes de Peace Plays édité par S. Lowe, avec notamment, The Celebration of Kokura de Berta Freistadt, Clam deDeborah Levy, Keeping Body and Soul Together de Stephen Lowe, The Fence par Common Ground, After the first death de Richard Stayton, End of the world with symposium to follow d'Arthur Kopit.

7. En témoignent ses Notebooks ainsi que ses recueils de poèmes, ou encore la pièce inachevée pour un groupe d'étudiants de l'université d'Essex : After the Assassinations (dans Plays, vol. 6).

8. En particulier dans son Commentary on TheWar Plays (1991).

9. Sur ce genre on se réfèrera à l'indépassable série en quatre volumes de la collection « Théâtre années 20 » : Le Théâtre d'agit-prop de 1917 à 1932 et leurs deux volumes de compléments : Le Théâtre d'intervention depuis 1968, ou encore, pour la période contemporaine en France, à O. Neveux.

10. Voir note 6

11. Sur ces images, voir M. Luecken.

12. P. Levi, p. 82-83.

13. «Si nous avions vécu nous ne nous serions pas satisfaits de vos stupidités barbares [...]/ Vous nous avez tués au nom de la liberté [...] / Quelle est la liberté que vous m'avez donnée ? / Deux poignées de cendres / Où est la liberté là-dedans? »

14. Commentary on The War Plays, p. 347.

15. G. Bataille, p. 74 .

16. «Je sais que c'est mal de dire des choses pareilles, mais qu'est-ce que je peux faire d'autre ?»

17. Bernard Faivre D'arcier, le directeur d'alors, organisait des événements consacrés à la guerre en Bosnie à chaque édition du Festival et avait été inscrit cette année-là sur l'éphémère « Liste Sarajevo » de Bernard-Henri Lévy aux élections européennes. L'édition suivante allait connaître une spectaculaire mobilisation au moment du massacre de l'enclave de Srebrenica. Sur cet engagement, voir E. Loyer et M. Baecque.

18. Mentionnons G. Lavaudant avec Lumières (qui incluait des scènes d'Agamemnon d'Eschyle), P. Sellars avec Les Perses d'Eschyle, J. Lassalle avec Andromaque d'Euripide, justement dans la Cour 
d'honneur du Palais des Papes cette année-là, et encore, avec d'autres formes de détours, S. Nordey avec Pylade de Pasolini, D.-G. Gabily avec Enfonçures, M. Deutsch avec Imprécations dans l'abattoir...

19. Didi-Hubermann oppose la prise de position, exemplaire dans les poèmes de la Kriegsfibel de Brecht, à la prise de parti, travers de l'art politique en général.

\section{RÉSUMÉS}

La trilogie théâtrale d'Edward Bond Pièces de guerre (1983-1985) constitue tant comme discours politique que comme geste esthétique, une proposition artistique originale d'approche dans une perspective progressiste de la question de la guerre nucléaire, thématique militante et culturelle cruciale durant ces dernières années de la guerre froide. Ces pièces naissent dans un contexte de forte mobilisation pacifiste, accompagnée d'une vive production culturelle, mais dont les perspectives politiques demeuraient limitées. Bond entendit s'opposer à ces modèles pour les dépasser. Chaque pièce organise son dispositif esthétique dans une perspective critique qui politise le discours en le déplaçant de l'indignation morale vers une analyse complexe de la position des individus-citoyens pris dans le chantage de "l'équilibre de la terreur ». Si ces pièces passèrent relativement inaperçues en Angleterre à l'époque, elles eurent une réelle portée en France dans les années 1990, dans un contexte politique radicalement changé, où elles trouvèrent à assumer les angoisses portées par les nouvelles guerres génocidaires.

Edward Bond's dramatic trilogy of The War Plays (1983-1985) are an original attempt to deal with the nuclear war issue, a major topic both for political activism and cultural creativity during the last years of the Cold War, precisely as a political statement as well as an aesthetic act. These plays were released during a massive peace movement, itself backed by a rich artistic production, which political views remained, though, limited to commonplaces. Bond intended to confront these models to go beyond them. Each of these plays sets its aesthetics according to a critical perspective allowing to politicize its statements, by moving from a protest on moral ground to a complex analysis of the single citizen's position trapped in the blackmail of the Balance of Terror. Poorly noticed at the time in England, these plays reached an audience in France in the 90s in a radically renewed political context when they came to express the fears carried by the new genocidal wars.

\section{INDEX}

Keywords : English Theater, Edward Bond, The War Plays, Cold War, Balance of Terror, United Kingdom, France

Mots-clés : Edward Bond, Pièces de guerre, théâtre anglais, équilibre de la terreur, RoyaumeUni, France 